\title{
Nonlocal Modification of Newtonian Gravity
}

\author{
Hans-Joachim Blome* \\ Department of Aerospace Technology, Aachen University of Applied Sciences, Hohenstaufenallee 6, 52064 Aachen, Germany
}

\author{
Carmen Chicone ${ }^{\dagger}$ \\ Department of Mathematics, University of Missouri, Columbia, Missouri 65211, USA
}

Friedrich W. Hehl ${ }^{\ddagger}$

Institute for Theoretical Physics, University of Cologne, 50923 Köln, Germany and

Department of Physics and Astronomy, University of Missouri, Columbia, Missouri 65211, USA

Bahram Mashhoon ${ }^{\S}$

Department of Physics and Astronomy, University of Missouri, Columbia, Missouri 65211, USA

(Dated: 10 March 2010, file ModNewton26.tex)

\begin{abstract}
The Newtonian regime of a recent nonlocal extension of general relativity (GR) is investigated. Nonlocality is introduced via a scalar "constitutive" kernel in a special case of the translational gauge theory of gravitation, namely, the teleparallel equivalent of GR. In this theory, the nonlocal aspect of gravity simulates dark matter. A nonlocal and nonlinear generalization of Poisson's equation of Newtonian gravitation is presented. The implications of nonlocality for the gravitational physics in the solar system are briefly studied.
\end{abstract}

PACS numbers: 03.30.+p, 04.50.Kd, 04.20.Cv, 11.10.Lm

Keywords: nonlocal gravity, general relativity, Newtonian gravity

\section{INTRODUCTION}

The Poisson equation of Newtonian gravitation,

$$
\nabla^{2} \Phi(t, \mathbf{x})=4 \pi G \rho(t, \mathbf{x})
$$

is a consequence of the inverse-square force law, which is ultimately based on solar-system observations that originally led to Kepler's laws of planetary motion. Einstein's gravitational field equations have generalized equation (1) into a consistent relativistic framework that is in good agreement with present solar-system data [1-3]. Nevertheless, on small laboratory scales, for instance, questions remain regarding the validity of the inverse-square law of gravitation and hence equation (1); at present, efforts continue on resolving such experimental problems [4-7]. This paper is about deviations from the inverse-square force law on galactic scales in order to resolve the problem of the flat rotation curves of spiral galaxies.

An essential component in the conceptual development of general relativity (GR) is the way Lorentz invariance is employed to describe what accelerated observers measure. Lorentz invariance is a fundamental symmetry and refers to measurements of ideal inertial observers that move uniformly forever on rectilinear timelike worldlines; therefore, an assumption is required to relate these ideal inertial observers to actual observers that are all non-

\footnotetext{
*Electronic address: blome@fh-aachen.de

${ }^{\dagger}$ Electronic address: chiconec@missouri.edu

‡Electronic address: hehl@thp.uni-koeln.de

$\S$ Electronic address: mashhoonb@missouri.edu
}

inertial (i.e., accelerated). The special theory of relativity uses the postulate of locality, namely, the assumption that an accelerated observer is pointwise inertial. The hypothesis of locality is known to be an approximation $[8,9]$; in fact, its domain of applicability is limited to motions with sufficiently low accelerations. The locality principle is also an essential ingredient of Einstein's heuristic principle of equivalence that is the cornerstone of general relativity. Nonlocal special relativity is a generalization of the standard theory that goes beyond the locality postulate and involves a certain average over the past worldline of the observer [10]. The principle of equivalence of inertial and gravitational masses implies a general connection between inertia and gravitation; therefore, one would expect that the nonlocality of accelerated observers in Minkowski spacetime would entail a nonlocal theory of gravitation [11]. However, a direct nonlocal generalization of GR has not been possible; that is, the highly local nature of Einstein's principle of equivalence apparently prevents a straightforward nonlocal generalization of GR. On the other hand, gauge theories of gravitation are in general less restrictive $[12,13]$; hence, in principle, a nonlocal generalization of GR can be constructed within the gauge approach to gravitation. Indeed, in recent papers $[14,15]$, a nonlocal generalization of Einstein's theory of gravitation has been presented on the basis of the teleparallel equivalent of GR [16]. In the simplest possibility, nonlocality is introduced via a scalar kernel. In this approach to nonlocal gravity, nonlocality can persist in the Newtonian limit of the theory.

To arrive at this limit in the linear approximation, it has been assumed, in addition, that the scalar kernel $\mathcal{K}(x, y)$ is a universal function of $x-y$ and $x$ is supposed 
to be in the future of $y$ to maintain causality $[14,15]$. In this case, the nonlocal gravitational field equations reduce to

$$
G_{\mu \nu}(x)+\int \mathcal{K}(x, y) G_{\mu \nu}(y) d^{4} y=\kappa T_{\mu \nu},
$$

cf. Eq. (62) of [15]. Here $G_{\mu \nu}$ is the linear Einstein tensor, $\kappa=8 \pi G / c^{4}$ and $T_{\mu \nu}$ is the energy-momentum tensor of matter $\left(\partial_{\nu} T^{\mu \nu}=0\right)$. In this gravitational background test particles and light rays respectively follow timelike and null geodesics of the metric tensor $g_{\mu \nu}=\eta_{\mu \nu}+h_{\mu \nu}$, where $\eta_{\mu \nu}$ is the Minkowski metric tensor given by $\operatorname{diag}(1,-1,-1,-1)$ and $h_{\mu \nu}$ is the linear perturbation away from flat spacetime. Greek indices run from 0 to 3 , while Latin indices run from 1 to 3 .

It is useful to move the nonlocal term to the right side of Eq. (2) via the Liouville-Neumann method of successive substitutions. Let us introduce iterated kernels $\mathcal{K}_{n}$ given by $\mathcal{K}_{1}(x, y)=\mathcal{K}(x, y)$ and

$$
\mathcal{K}_{n+1}(x, y)=\int \mathcal{K}(x, z) \mathcal{K}_{n}(z, y) d^{4} z .
$$

Inspection of Eq. (3) reveals that in each iterated kernel $\mathcal{K}_{n}(x, y)$, with $n>1$, causality is preserved so that $x$ is in the future of $y$, but in general $\mathcal{K}_{n}(x, y)$ is no longer a function of $x-y$. This is therefore the case for the reciprocal kernel $\mathcal{R}(x, y)$ as well,

$$
-\mathcal{R}(x, y)=\sum_{n=1}^{\infty} \mathcal{K}_{n}(x, y) .
$$

Thus Eq. (2) can be written as

$$
G_{\mu \nu}(x)=\kappa\left[T_{\mu \nu}(x)+\int \mathcal{R}(x, y) T_{\mu \nu}(y) d^{4} y\right],
$$

so that the nonlocal theory in this approximation is equivalent to GR but with an additional source term. In fact, the nonlocal aspect of gravity can appear as dark matter given by the integral transform of $T_{\mu \nu}$ by the causal reciprocal kernel $\mathcal{R}$. In this paper we take the view that $\mathcal{R}$ must be determined from observation; for instance, lensing observations of colliding clusters of galaxies - such as in the case of the Bullet Cluster [17] could provide clues regarding the nature of the full timedependent reciprocal kernel (cf. Section III).

In the Newtonian limit $(c \rightarrow \infty)$, retardation effects can be neglected and hence we can assume that each iterated kernel in Eq. (3) is proportional to $\delta\left(x^{0}-y^{0}\right)$. It then follows from Eq. (3) that

$$
\mathcal{R}(x, y)=\delta\left(x^{0}-y^{0}\right) q(\mathbf{x}-\mathbf{y}),
$$

where $q$ is the spatial convolution kernel. Using this limiting form of the kernel in Eq. (5), we find in the Newtonian limit the nonlocal Poisson equation $[14,15]$

$$
\nabla^{2} \Phi=4 \pi G\left[\rho(t, \mathbf{x})+\rho_{\mathrm{D}}(t, \mathbf{x})\right]
$$

where the "density of dark matter" $\rho_{\mathrm{D}}$ is given by

$$
\rho_{\mathrm{D}}(t, \mathbf{x})=\int q(\mathbf{x}-\mathbf{y}) \rho(t, \mathbf{y}) d^{3} y .
$$

Here $q$ is a universal function that is independent of the nature of the source $[14,15]$. This simplifying assumption is relaxed in Section II, where we discuss the general form of the nonlocal kernel in the Newtonian limit.

This paper is based on the assumption that there is no actual dark matter. According to the approximation scheme employed in [14, 15], the nonlocal aspect of the gravitational interaction acts like dark matter of density $\rho_{\mathrm{D}}$ that is linearly related to the actual matter density via the kernel $q$ as in equation (8). Consider, for example, the circular motion of stars in the disk of a spiral galaxy in connection with the observed flat rotation curves in such galaxies (see, for instance, [18, 19] and references therein). At radius $r$ outside the bulge, the Newtonian acceleration of gravity for such a star is nearly $v_{0}^{2} / r$, where $v_{0}$ is a constant speed. Poisson's equation then implies that the corresponding density of "dark" matter must be $v_{0}^{2} /\left(4 \pi G r^{2}\right)$. Extending this $\rho_{\mathrm{D}}$ to a spherical distribution of "dark" matter by assumption, the result can be compared with Eq. (8): neglecting the extended nature of the galactic bulge and setting $\rho(t, \mathbf{y})=M \delta(\mathbf{y})$, where $M$ is the effective galactic mass, we find

$$
q(\mathbf{x}-\mathbf{y})=\frac{1}{4 \pi \lambda} \frac{1}{|\mathbf{x}-\mathbf{y}|^{2}},
$$

where $\lambda=G M / v_{0}^{2}$ is of the order of $1 \mathrm{kpc}$. The universality of the nonlocal kernel implies that $\lambda$ must be a constant and hence $M \propto v_{0}^{2}$. The resulting nonlocal modification of Poisson's equation (7)-(9) has been previously discussed in connection with the Tohline-Kuhn scheme [20-22]. In particular, for a point source $\rho(t, \mathbf{x})=M \delta(\mathbf{x})$, Eqs. (7)-(9) imply that

$$
\Phi(t, \mathbf{x})=-\frac{G M}{|\mathbf{x}|}+\frac{G M}{\lambda} \ln \left(\frac{|\mathbf{x}|}{\lambda}\right) .
$$

This coincides with Tohline's original suggestion regarding a modification of Newton's law of gravitation in order to account for the flat rotation curves of spiral galaxies [20]. A lucid and enlightening account of the TohlineKuhn approach is contained in the review paper of Bekenstein [22].

It is clear from this brief account that, as shown in detail in $[14,15]$, the Tohline-Kuhn extension of Newtonian gravitation to the realm of galaxies can be naturally embedded within a nonlocal generalization of GR. However, the Tohline-Kuhn scheme disagrees with the empirical Tully-Fisher law [23]. The Tully-Fisher relation involves a correlation between the luminosity of a spiral galaxy and the corresponding asymptotic speed $v_{0}$. This relation, combined with other empirical data regarding massto-light ratio, roughly favors $M \propto v_{0}^{4}$, instead of $M \propto v_{0}^{2}$ that follows from the Tohline-Kuhn scheme. Various aspects of this issue have been discussed in $[22,24,25]$. To 
go beyond the Tohline-Kuhn scheme, we discuss a generalization of Eqs. (7)-(8) in Section II, where the Newtonian limit of nonlocal gravity is discussed in detail. It is hoped that this more general treatment of the Newtonian limit could help in the resolution of the discrepancy with the Tully-Fisher law.

\section{NEWTONIAN LIMIT OF NONLOCAL GRAVITY}

The linear approximation in nonlocal gravity involves a linear perturbation away from Minkowski spacetime. Consider a background Minkowski spacetime with global inertial coordinates $x^{\alpha}=(c t, \mathbf{x})$. The gravitational potentials are given by the tetrad field $e_{\mu}{ }^{\nu}(x)$ such that

$$
e_{\mu}^{\alpha}=\delta_{\mu}^{\alpha}+\psi^{\alpha}{ }_{\mu}, \quad e^{\mu}{ }_{\alpha}=\delta_{\alpha}^{\mu}-\psi^{\mu}{ }_{\alpha},
$$

where $\psi_{\mu \nu}$ is proportional to $G / c^{2}$. The indices are raised and lowered by means of the Minkowski metric tensor $\eta_{\alpha \beta}$. The gravitational field strength is then given by

$$
C_{\mu \nu \rho}=\psi_{\rho \nu, \mu}-\psi_{\rho \mu, \nu}
$$

We define the modified field strength $\mathfrak{C}_{\mu \nu \rho}$ via

$$
\begin{aligned}
\mathfrak{C}_{\mu \nu \rho}=C_{\mu \nu \rho}+\psi_{[\mu \nu], \rho} & +\eta_{\mu \rho}\left(\psi_{, \nu}-\psi_{\sigma \nu,}{ }^{\sigma}\right) \\
& -\eta_{\nu \rho}\left(\psi_{, \mu}-\psi_{\sigma \mu}{ }^{\sigma}{ }^{\sigma}\right),
\end{aligned}
$$

where $\psi=\eta_{\alpha \beta} \psi^{\alpha \beta}$. Thus both $C_{\mu \nu \rho}$ and $\mathfrak{C}_{\mu \nu \rho}$ are antisymmetric in their first two indices. The coordinate components of the metric tensor are given by

$$
g_{\mu \nu}=\eta_{\alpha \beta} e_{\mu}^{\alpha} e_{\nu}^{\beta}=\eta_{\mu \nu}+\psi_{\mu \nu}+\psi_{\nu \mu}
$$

The nonlocal gravitational field equations in this linear approximation scheme then reduce to $[14,15]$

$$
G_{\mu \nu}(x)+\eta^{\rho \sigma} \int \frac{\partial K(x, y)}{\partial x^{\rho}} \mathfrak{C}_{\mu \sigma \nu}(y) d^{4} y=\kappa T_{\nu \mu}(x)
$$

where $G_{\mu \nu}$ is the linearized Einstein tensor in terms of $g_{\mu \nu}, T_{\mu \nu}$ is the energy-momentum tensor of mass-energy in Minkowski spacetime and $\partial_{\mu} T^{\nu \mu}=0$. Equation (15) corresponds to Eq. (60) of [15]. If $K(x, y)=\mathcal{K}(x, y)$, then Eq. (15) reduces to Eq. (2), since $G^{\mu \nu}=\partial_{\sigma} \mathfrak{C}^{\mu \sigma \nu}$. In this case, $T_{\mu \nu}$ must be symmetric and hence the antisymmetric part of $\psi_{\mu \nu}$ does not participate in gravitational dynamics and can thus be neglected. In general, however, the energy-momentum tensor is not symmetric; therefore, Eq. (15) contains sixteen field equations for the sixteen components of $\psi_{\mu \nu}$.

The kernel $K(x, y)$ could in general depend upon the structure of the source in a manner that is consistent with the linear approximation scheme. This situation is considered in this section for the nonlocal modification of Newtonian gravity. However, in previous work [14, 15], this possibility was neglected for the sake of simplicity and it was assumed instead that $K(x, y)$ is some universal function of $x-y$.

To approach the Newtonian limit of the nonlocal theory, we tentatively assume that the dynamics in Eq. (15) is dominated by the Newtonian potential $\Phi$ such that

$$
\psi_{00}=\psi_{11}=\psi_{22}=\psi_{33}=\frac{1}{c^{2}} \Phi
$$

and $\psi=-2 \Phi / c^{2}$, while the other components of $\psi_{\mu \nu}$ may be neglected in the Newtonian limit. This assumption corresponds to the circumstance that as in GR, one expects that the main effects would be associated with a diagonal spacetime metric of the form $g_{\mu \nu}=\eta_{\mu \nu}+h_{\mu \nu}$, where $h_{\mu \nu}=2 c^{-2} \Phi \operatorname{diag}(1,1,1,1)$. In this case, we find from Eq. (13) that

$$
c^{2} \mathfrak{C}_{0 j 0}=-2 \partial_{j} \Phi
$$

moreover, as in GR, $c^{2} G_{00}=2 \nabla^{2} \Phi$. Hence, with $T_{00}=$ $\rho c^{2}$ and

$$
K(x, y)=\delta\left(x^{0}-y^{0}\right) k(\mathbf{x}, \mathbf{y})
$$

where retardation effects have been neglected, the Newtonian limit of Eq. (15) is of the form

$$
\nabla^{2} \Phi(\mathbf{x})+\sum_{i} \int \frac{\partial k(\mathbf{x}, \mathbf{y})}{\partial x^{i}} \frac{\partial \Phi(\mathbf{y})}{\partial y^{i}} d^{3} y=4 \pi G \rho(\mathbf{x})
$$

This is a more general form of equations (7) and (8); furthermore, for simplicity we have suppressed any temporal dependence. Equation (19) reduces to Eqs. (7)-(8) if $k(\mathbf{x}, \mathbf{y})=k^{\prime}(\mathbf{x}-\mathbf{y})$, for which the reciprocal kernel is $q(\mathbf{x}-\mathbf{y})$; however, as pointed out in $[14,15]$, the kernel could in general depend upon the Weitzenböck invariants at $x$ and $y$. For the case under consideration, these are

$$
\begin{aligned}
c^{4} C^{\mu \nu \rho} C_{\mu \nu \rho} & =6 \eta^{\alpha \beta} \frac{\partial \Phi}{\partial x^{\alpha}} \frac{\partial \Phi}{\partial x^{\beta}} \\
c^{4} C^{\mu \nu \rho} C_{\rho \nu \mu} & =3 \eta^{\alpha \beta} \frac{\partial \Phi}{\partial x^{\alpha}} \frac{\partial \Phi}{\partial x^{\beta}} \\
c^{4} C^{\mu \nu}{ }_{\nu} C_{\mu \rho}{ }^{\rho} & =9\left(\frac{\partial \Phi}{\partial x^{0}}\right)^{2}-(\nabla \Phi)^{2} .
\end{aligned}
$$

It follows that in the limiting case $(c \rightarrow \infty)$ under consideration, the Weitzenböck invariants all reduce to the square of $|\nabla \Phi|$, which is the magnitude of the Newtonian gravitational acceleration. Hence, one may express the kernel as

$$
k(\mathbf{x}, \mathbf{y})=k^{\prime}(\mathbf{x}-\mathbf{y})+k^{\prime \prime}\left(\mathbf{x}-\mathbf{y} ; \frac{\left|\nabla_{\mathbf{y}} \Phi\right|}{\left|\nabla_{\mathbf{x}} \Phi\right|}\right),
$$

so that $k(\mathbf{x}, \mathbf{y})$ depends on the structure of the source, but is otherwise consistent with the linear approximation scheme.

Equations (19) and (23) imply that

$$
\nabla_{\mathbf{x}}^{2} \Phi+\int k^{\prime}(\mathbf{x}, \mathbf{y}) \nabla_{\mathbf{y}}^{2} \Phi d^{3} y=4 \pi G\left(\rho+\rho_{\Phi}\right),
$$


where $\rho_{\Phi}$ is defined by

$$
\rho_{\Phi}(\mathbf{x})=-\frac{1}{4 \pi G} \sum_{i} \int \frac{\partial k^{\prime \prime}}{\partial x^{i}} \frac{\partial \Phi}{\partial y^{i}} d^{3} y .
$$

We recall that $q(\mathbf{x}-\mathbf{y})$ is reciprocal to $k^{\prime}(\mathbf{x}-\mathbf{y})$; therefore,

$$
\nabla^{2} \Phi=4 \pi G\left\{\rho+\rho_{\Phi}+\int q(\mathbf{x}-\mathbf{y})\left[\rho(\mathbf{y})+\rho_{\Phi}(\mathbf{y})\right] d^{3} y\right\} .
$$

In the absence of $\rho_{\Phi}$, Eq. (26) is equivalent to equations (7) and (8). However, Eqs. (24)-(26) contain a more general treatment of the Newtonian limit of the nonlocal theory. Such a treatment is necessary in order to help resolve observational problems associated with the empirical Tully-Fisher relation [23].

Equation (26) is a nonlinear integro-differential relation for the Newtonian potential $\Phi$. It is clear from Eq. (23) that scaling $\Phi$ by a constant factor leaves the kernel invariant. Thus $\Phi$ given by Eq. (26), despite the nonlinearity of this equation, will be linear in the gravitational constant $G$, as would be expected on physical grounds. Moreover, as in Newton's theory, the potential $\Phi$ can be determined from the modified Poisson equation only up to an additive constant. Solutions of equations (19) and (23), or equivalently Eq. (26), are not known at present; therefore, in the following sections we resort to the discussion of the solutions of the linear part of the modified Poisson equation.

\section{ORIGIN OF KERNEL $q$}

We return to the study of equations (7) and (8). The main feature of these equations is the existence of a linear relation between the potential $\Phi$ and matter density $\rho$; that is,

$$
\Phi(t, \mathbf{x})=G \int \chi(\mathbf{x}, \mathbf{y}) \rho(t, \mathbf{y}) d^{3} y .
$$

The Green function $\chi$ can in this case be simply obtained from Eq. (10), namely, $\chi$ is a function of $|\mathbf{x}-\mathbf{y}|$ and is given by

$$
\chi(\mathbf{x}, \mathbf{y})=-\frac{1}{|\mathbf{x}-\mathbf{y}|}+\frac{1}{\lambda} \ln \left(\frac{|\mathbf{x}-\mathbf{y}|}{\lambda}\right) .
$$

It can be easily verified that $\chi$ is a solution of

$$
\nabla_{\mathbf{x}}^{2} \chi(\mathbf{x}, \mathbf{y})=4 \pi[\delta(\mathbf{x}-\mathbf{y})+q(\mathbf{x}-\mathbf{y})] .
$$

One can develop potential theory (see, for instance, [26]) for nonlocal gravity on the basis of equations (27)-(28). Moreover, the force of gravity per unit test mass is given by

$$
-\nabla \Phi=-G \int\left[\frac{\mathbf{x}-\mathbf{y}}{|\mathbf{x}-\mathbf{y}|^{3}}+\frac{1}{\lambda} \frac{\mathbf{x}-\mathbf{y}}{|\mathbf{x}-\mathbf{y}|^{2}}\right] \rho(t, \mathbf{y}) d^{3} y .
$$

The integral form of equation (7) - as well as its generalization in equation (26) - can be obtained using Green's theorem; this is the subject of Appendix A.

In this paper, we take the tentative view that $q(\mathbf{r})$ must ultimately be determined via observation. That is, this nonlocal "Newtonian" aspect of gravity, just as the local Newtonian inverse-square force law, is a feature of the gravitational interaction deducible from experience. Thus there is no fundamental basis at present for the determination of the specific form of the nonlocal kernel other than the concordance of equation (7) with observational data. As pointed out in [15], the convolution theorem for Fourier integrals may be employed to determine $q$ using Eq. (8) once $\rho$ and $\rho_{\mathrm{D}}$ are completely known. However, this expectation is unrealistic at present. Since Newton's time, various modifications of the inverse-square force law have been contemplated [27]; similarly, we can investigate how the potential (10) would change if the kernel (9) is modified.

Let us first consider a kernel of the form

$$
q(\mathbf{r})=\frac{1}{4 \pi \lambda} \frac{1}{r^{2}+\ell_{0}^{2}}
$$

where $r=|\mathbf{r}|$ and $\ell_{0}$ is a constant length parameter such that for $\ell_{0} \neq 0$, Eq. (31) is, unlike Eq. (9), singularity-free. Integrating the corresponding Eq. (29), it is straightforward to show that the analog of Eq. (10) is in this case

$\Phi=-\frac{G M}{r}+\frac{G M}{\lambda}\left[\ln \left(\frac{\left(r^{2}+\ell_{0}^{2}\right)^{1 / 2}}{\lambda}\right)+\frac{\ell_{0}}{r} \tan ^{-1}\left(\frac{r}{\ell_{0}}\right)\right]$,

which reduces to Eq. (10) for $\ell_{0}=0$. We note that the term in square brackets goes to $1+\ln \left(\ell_{0} / \lambda\right)$ for $r \rightarrow$ 0 ; therefore, the logarithmic singularity in Eq. (10) is avoided by the introduction of $\ell_{0} \neq 0$. Next, let

$$
q(\mathbf{r})=\frac{1}{4 \pi \lambda} \frac{1}{r^{2}} e^{-r / L_{0}},
$$

where $L_{0}$ is a constant length that renders the integral of Eq. (33) finite over all space. This is necessary to ensure that the total mass of "dark" matter is finite; as pointed out in [15], the total "dark matter mass" is infinite if equation (9) is taken to be valid for $|\mathbf{x}-\mathbf{y}| \rightarrow \infty$. But empirical data are not available beyond galaxy clusters and it is rather likely that Eq. (9) must be modified for sufficiently large $|\mathbf{x}-\mathbf{y}|$. As before, it is possible to integrate equation (29) in this case and the result is

$$
\begin{aligned}
\Phi= & -\frac{G M}{r}+\frac{G M}{\lambda}\left[1+\frac{L_{0}}{r}\left(e^{-r / L_{0}}-1\right)\right. \\
& \left.+\operatorname{Ei}\left(-\frac{r}{L_{0}}\right)-C-\ln \left(\frac{\lambda}{L_{0}}\right)\right],
\end{aligned}
$$

which reduces to Eq. (10) for $L_{0}=\infty$. Here $C=$ $0.577 \ldots$ is the Euler constant and we use for the exponential integral function $\mathrm{Ei}(x)$ the expression

$$
\operatorname{Ei}(-x)=C+\ln x+\sum_{n=1}^{\infty} \frac{(-1)^{n} x^{n}}{n \cdot n !}, \quad x>0
$$


and the asymptotic expansion

$$
\operatorname{Ei}(-x)=e^{-x} \sum_{n=1}^{\infty}(-1)^{n} \frac{(n-1) !}{x^{n}}, \quad x \rightarrow \infty ;
$$

see the first formula in (8.214) and formula (8.125) on page 927 of Ref. [28]. It is important to note that for $r \rightarrow \infty, \Phi$ has a constant value in this case.

It is clear from these considerations that variations in the simple form of the kernel (9) can lead to complicated expressions for the gravitational potential. It is therefore interesting to consider possible unique characterizations of this kernel. In this connection, we recall that in Newton's theory, the exterior gravitational potential of a point mass is proportional to $1 / r$ and satisfies Laplace's equation. In fact, the fundamental harmonic solution of Laplace's equation in $n$-dimensional Euclidean space is $1 / r^{n-2}$ for $n>2$ and $\ln r$ for $n=2$. In the $n=4$ case, this result has a natural analog in Minkowski spacetime with inertial coordinates $(c t, x, y, z)$, namely, $\square W=0$, where

$$
W^{-1}=-c^{2}\left(t-t_{0}\right)^{2}+\left(x-x_{0}\right)^{2}+\left(y-y_{0}\right)^{2}+\left(z-z_{0}\right)^{2} .
$$

Here $\square$ is the d'Alembertian operator defined by $\square=$ $-\eta^{\alpha \beta} \partial_{\alpha} \partial_{\beta}$. For an interesting discussion of such solutions and their singularities, see chapter IX of Synge [29].

Let us note, for instance, that Eq. (9) satisfies

$$
\nabla^{2} q=8 \pi \lambda q^{2}
$$

That is, up to a constant factor, $q(\mathbf{r})$ is a timeindependent solution of the semilinear wave equation [30]

$$
\square \varphi=\varphi^{2} .
$$

It is demonstrated in Appendix B that there is a oneparameter family of nonzero spherically symmetric solutions of Eq. (38) that vanishes together with all of their derivatives as $r \rightarrow \infty$. These solutions, as discussed in detail in Appendix B, behave as

$$
\frac{1}{4 \pi \lambda}\left(\frac{1}{r^{2}} \pm \frac{C_{0}}{r^{2+\sigma}}\right)
$$

for $r \rightarrow \infty$, where $\sigma=(\sqrt{17}-3) / 2$ and $C_{0}$ is an arbitrary constant parameter. With a suitable choice of $C_{0}$, these latter solutions would also be consistent with galactic data.

Equations (38) and (39) make it possible to contemplate appropriate generalizations of Eq. (9). For instance, the invariance of Eq. (38) under spatial translations indicates that

$$
\frac{1}{4 \pi \lambda} \frac{1}{\left(x-x_{0}\right)^{2}+\left(y-y_{0}\right)^{2}+\left(z-z_{0}\right)^{2}}
$$

is also a solution of Eq. (38) that reduces to $q(\mathbf{r}), \mathbf{r}=$ $(x, y, z)$, for $\mathbf{r}_{0}=0$. Furthermore, it follows in a similar way from the scalar field equation (39) that timedependent kernels can be constructed via Lorentz transformations. Consider, for instance, a pure boost in the $x$ direction with speed $v$; then,

$$
\frac{1}{4 \pi \lambda} \frac{1}{\gamma^{2}(x+v t)^{2}+y^{2}+z^{2}}
$$

is a solution of Eq. (39) that reduces to $q(\mathbf{r})$ for $v=0$. Here $\gamma$ is the Lorentz factor corresponding to speed $v$. This means that one could construct reciprocal kernels involving two events $(c t, \mathbf{r})$ and $\left(c t^{\prime}, \mathbf{r}^{\prime}\right)$ using functions of the form

$$
\frac{1}{4 \pi \lambda} \frac{1}{\gamma^{2}\left[\left(x-x^{\prime}\right)+v\left(t-t^{\prime}\right)\right]^{2}+\left(y-y^{\prime}\right)^{2}+\left(z-z^{\prime}\right)^{2}}
$$

together with an appropriate causal ordering of the events.

In the rest of the paper, we simply employ kernel (9).

\section{EXTENDED SPHERICAL SOURCE}

According to the inverse-square force law, a homogeneous spherical distribution of matter attracts an external particle as if the mass of the sphere were concentrated at its center. However, this important result of Newtonian gravitation would no longer hold in general with a modified force law. To illustrate this point, equations (27) and (28) can be used to evaluate the exterior gravitational potential for any source distribution. Specifically, let us consider a spherically symmetric mass distribution of radius $R_{0}$ such that

$$
M=4 \pi \int_{0}^{R_{0}} \rho(r) r^{2} d r
$$

At a spacetime position $(t, \mathbf{X})$ exterior to the static source, $R=|\mathbf{X}|>R_{0}$,

$$
\Phi(t, \mathbf{X})=-\frac{G M}{R}+\frac{2 \pi G}{\lambda} \int_{0}^{R_{0}} F(R, r) \rho(r) r^{2} d r .
$$

Here, the first term is due to the fact that in Newtonian gravitation the exterior potential of any spherically symmetric distribution can be replaced at its center by a point source, whose mass is equal to the total mass of the spherical distribution. Moreover, in Eq. (45), $F(R, r)$ is given by

$$
F(R, r)=\int_{0}^{\pi} \ln \left(\frac{\left(R^{2}+r^{2}-2 R r \cos \theta\right)^{1 / 2}}{\lambda}\right) \sin \theta d \theta,
$$

since the symmetry of the configuration makes it possible to choose the $\mathrm{z}$ axis to be along the position vector $\mathbf{X}$. It is straightforward to show that

$$
F(R, r)=2 \ln \left(\frac{R}{\lambda}\right)+f\left(\frac{r}{R}\right),
$$

where for $\epsilon=r / R<1$,

$$
f(\epsilon)=-1+\frac{1}{2 \epsilon}\left[(1+\epsilon)^{2} \ln (1+\epsilon)-(1-\epsilon)^{2} \ln (1-\epsilon)\right] .
$$


Thus Eq. (45) can be written as

$$
\begin{aligned}
\Phi(t, \mathbf{X})= & -\frac{G M}{R}+\frac{G M}{\lambda} \ln \left(\frac{R}{\lambda}\right) \\
& +\frac{2 \pi G}{\lambda} \int_{0}^{R_{0}} f\left(\frac{r}{R}\right) \rho(r) r^{2} d r,
\end{aligned}
$$

which is the sum of the contribution of a point mass $M$ as in Eq. (10) and an extra term due to the extension of the source. One can show that

$$
f(\epsilon)=\frac{1}{3} \epsilon^{2}+\frac{1}{30} \epsilon^{4}+O\left(\epsilon^{6}\right)
$$

using the following relation that is valid for $|x|<1$,

$$
\ln (1+x)=\sum_{n=1}^{\infty}(-1)^{n+1} \frac{x^{n}}{n} .
$$

To get an explicit result, let us assume, for the sake of simplicity, that $\rho(r)=\rho_{0}$ is a constant. Then, the integral in Eq. (49) can be evaluated analytically using the formulas (2.729) on page 205 of Ref. [28]. In any case, the dominant terms can also be calculated directly from Eq. (50) and the end result is

$$
\begin{aligned}
\Phi= & -\frac{G M}{R}+\frac{G M}{\lambda} \ln \left(\frac{R}{\lambda}\right) \\
& +\frac{G M}{10 \lambda}\left(\frac{R_{0}}{R}\right)^{2}\left[1+\frac{1}{14}\left(\frac{R_{0}}{R}\right)^{2}+\cdots\right] .
\end{aligned}
$$

The force of gravity per unit test mass is conservative and is given by $-\nabla \Phi$; for Eq. (52), this points in the direction of the source and has a magnitude

$$
\frac{d \Phi}{d R}=\frac{G M}{R^{2}}+\frac{G M}{\lambda R}\left[1-\frac{1}{5}\left(\frac{R_{0}}{R}\right)^{2}-\frac{1}{35}\left(\frac{R_{0}}{R}\right)^{4} \cdots\right] .
$$

Here, the quantity in brackets is close to unity, since $R>$ $R_{0}$; therefore, we may conclude that the extended form of a nearly homogeneous spherical source does not significantly alter the main physical results of the Tohline-Kuhn scheme.

\section{SOLAR-SYSTEM EFFECTS}

It is interesting to search for evidence of nonlocal gravity in the solar system. As a first step in this endeavor, let us consider gravitational physics in the solar system using a Tohline-Kuhn gravitational potential of the form

$$
\Phi=-\frac{G M}{r}+\frac{G M}{\lambda} \ln \frac{r}{\lambda^{\prime}},
$$

where the gravitational source is at the origin of coordinates. Here $\lambda^{\prime}$ is assumed to be a galactic-scale length. For $\lambda=10 \mathrm{kpc}, 2$ A.U. $/ \lambda \approx 10^{-9}$, hence the logarithmic term in Eq. (54) is expected to be a very small perturbation of the Newtonian potential. The following preliminary considerations are based on the fact that in the nonlocal generalization of GR under consideration here, light rays and test particles move along null and timelike geodesics, respectively.

\section{A. Time Delay}

In Newtonian gravity the potential vanishes at infinity by convention. However, the logarithmic term in Eq. (54) is assumed to vanish at the radial distance $\lambda^{\prime}$. While this is of no consequence in Newtonian gravity, it matters here as the spacetime interval depends on $\Phi$. Consider, for instance, the gravitational time delay $\Delta$ between events $P_{1}:\left(c t_{1}, \mathbf{r}_{1}\right)$ and $P_{2}:\left(c t_{2}, \mathbf{r}_{2}\right)$ when a light signal travels from $P_{1}$ to $P_{2}$. Let $L=\left|\mathbf{r}_{2}-\mathbf{r}_{1}\right|$ and $\ell: 0 \rightarrow L$ be the distance along a straight line from $P_{1}$ to $P_{2}$; then, $\Delta=t_{2}-\left(t_{1}+L / c\right)$ is given by [31]

$$
\Delta=-\frac{2}{c^{3}} \int_{P_{1}}^{P_{2}} \Phi d \ell .
$$

It follows from a detailed calculation that

$$
\begin{aligned}
\Delta= & \frac{2 G M}{c^{3}} \ln \frac{r_{2}+\hat{\mathbf{n}} \cdot \mathbf{r}_{2}}{r_{1}+\hat{\mathbf{n}} \cdot \mathbf{r}_{1}} \\
& -\frac{2 G M}{c^{3} \lambda}\left\{\left(\hat{\mathbf{n}} \cdot \mathbf{r}_{2}\right) \ln \frac{r_{2}}{\lambda^{\prime}}-\left(\hat{\mathbf{n}} \cdot \mathbf{r}_{1}\right) \ln \frac{r_{1}}{\lambda^{\prime}}-L\right. \\
& \left.+A\left[\tan ^{-1}\left(\frac{\hat{\mathbf{n}} \cdot \mathbf{r}_{2}}{A}\right)-\tan ^{-1}\left(\frac{\hat{\mathbf{n}} \cdot \mathbf{r}_{1}}{A}\right)\right]\right\} .
\end{aligned}
$$

Here

$$
\hat{\mathbf{n}}=\frac{1}{L}\left(\mathbf{r}_{2}-\mathbf{r}_{1}\right), \quad A=\frac{1}{L}\left|\mathbf{r}_{1} \times \mathbf{r}_{2}\right| .
$$

The net result is a sum of the Shapiro time delay together with the contribution of the logarithmic term in the potential. The relative magnitude of these delays is expected to be similar as in Eq. (54).

In the rest of this section, only the derivative of the potential is involved; therefore, $\lambda^{\prime}$ drops out of our calculations. For instance, in the gravitational shift of the frequency of light only the difference in the potential $\Phi$ at two spatially separated events is significant.

\section{B. Deflection of Light}

The net deflection angle $D$ of a light ray due to a point mass $M$ with potential $\Phi$ is given by twice the Newtonian expectation in the first post-Newtonian approximation, which for Eq. (54) works out to be

$$
D=\frac{4 G M}{c^{2} \zeta}+\frac{2 \pi G M}{c^{2} \lambda}
$$

where $\zeta$ is the distance of the closest approach. The bending angle is thus slightly larger than the Einstein 
angle by a constant. However, the extra deflection is not expected to remain constant for an extended source (cf. Section IV).

The effect of the logarithmic potential is $\sim 10^{-12}$ of the Einstein angle for the bending of light by the Sun.

\section{Perihelion Precession}

The gravitational force due to potential (54) is radial and conservative. Therefore, the perturbing influence of the logarithmic term in Eq. (54) on Keplerian orbits is such that the orbit remains planar and the orbital angular momentum is unchanged. Let $(r, \phi)$ be polar coordinates in the orbital plane and consider an unperturbed Keplerian ellipse given by

$$
r=\frac{a\left(1-e^{2}\right)}{1+e \cos \hat{\phi}}
$$

where $a$ is the semimajor axis of the ellipse, $e$ is its eccentricity, $\hat{\phi}=\phi-g$ and $g$ is the argument of the pericenter. Under the influence of the radial perturbing acceleration $-(G M / \lambda) r^{-1}$, the orbital elements of the osculating ellipse vary in accordance with the Lagrange planetary equations [32]. In this case, we find

$$
\begin{aligned}
& \frac{d a}{d t}=-\frac{2 \omega a^{2} e}{\lambda\left(1-e^{2}\right)^{3 / 2}}(1+e \cos \hat{\phi}) \sin \hat{\phi}, \\
& \frac{d g}{d t}=\frac{\omega a}{\lambda e\left(1-e^{2}\right)^{1 / 2}}(1+e \cos \hat{\phi}) \cos \hat{\phi},
\end{aligned}
$$

where $\omega$ is the Keplerian frequency of the osculating ellipse $\left(\omega^{2}=G M / a^{3}\right)$. Moreover, $\left[G M a\left(1-e^{2}\right)\right]^{1 / 2}$ is the magnitude of the specific orbital angular momentum and remains constant. Let us note here that only positive square roots are considered in this paper.

The elements of the osculating ellipse change slowly according to Eqs. (60) and (61); therefore, it is natural to average the right-hand sides of these equations over the fast orbital motion with period $T=2 \pi / \omega$. That is, we define the average of a quantity $Q$ to be

$$
<Q>\frac{1}{T} \int_{0}^{T} Q d t
$$

so that

$$
<Q>=\frac{\left(1-e^{2}\right)^{3 / 2}}{2 \pi} \int_{0}^{2 \pi} \frac{Q d \phi}{(1+e \cos \hat{\phi})^{2}},
$$

since $r^{2} d \phi / d t=\left[G M a\left(1-e^{2}\right)\right]^{1 / 2}$ for the unperturbed orbit. It follows that $\langle d a / d t\rangle=0$, so that the semimajor axis remains unchanged on the average. This is also the case for the orbital eccentricity due to the constancy of the angular momentum. Thus the ellipse keeps its shape on the average but precesses, since

$$
<\frac{d g}{d t}>=-\frac{\omega a}{2 \lambda} P(e),
$$

where

$$
P(e)=\frac{2}{e^{2}}\left[\left(1-e^{2}\right)^{1 / 2}-\left(1-e^{2}\right)\right]
$$

decreases from unity at $e=0$ to zero at $e=1$. Here we have used the fact that

$$
\int_{\alpha_{0}}^{\alpha_{0}+2 \pi} \frac{d \alpha}{1+e \cos \alpha}=\frac{2 \pi}{\left(1-e^{2}\right)^{1 / 2}} .
$$

One can obtain the same result for the pericenter precession frequency from the study of the variation of the Runge-Lenz vector [33].

For the solar system, the resulting perihelion precession [34] is retrograde and for $\lambda=10 \mathrm{kpc}$, it is about $10^{-3}$ of Einstein's value for Mercury and about $2 \times 10^{-2}$ for Earth, as there is more "dark matter" to influence the outer orbits. The general relativistic contribution to the perihelion precession of Mercury is known at present at the level of about one part in a thousand; therefore, the possible contribution of the logarithmic potential is hidden within the present measurement error. Future improvements in such measurements may make it possible to detect the influence of nonlocal gravity in the solar system. Nevertheless nonlocal effects appear at present to be too small to be detectable. For instance, the contribution of the logarithmic term to the Pioneer anomaly is $\sim 10^{-4}$ of the anomalous acceleration of the Pioneer spacecraft.

It appears that other anomalies in the solar systemsuch as the flyby anomaly, the possible secular increase in the Astronomical Unit and the increase in the eccentricity of Moon's orbit (see Ref. [35]) — are not directly affected by the conservative perturbing force under consideration here. We should also mention that solar-system deviations from GR can in principle be used to place lower bounds on the constant lengthscales that appear in the logarithmic term in Eq. (54).

\section{DISCUSSION}

Starting from first principles, arguments have been advanced for a nonlocal generalization of Einstein's theory of gravitation $[10,11,14,15]$. In such a theory, the gravitational field is local, but satisfies nonlocal integrodifferential field equations. These are obtained from the local field equations via a nonlocal "constitutive" ansatz, as described in Appendix $\mathrm{C}$ within the general context of gauge theories of gravitation that are less restrictive than GR and thus make it possible to implement this procedure.

The Newtonian limit of the simplest nonlocal GR theory involving a scalar constitutive kernel [14, 15] is studied in this paper. It is shown that the theory reduces to a nonlinear and nonlocal modification of Poisson's equation of Newtonian gravity. The exploration of the nonlinear aspects of this equation is beyond the scope of the 
present work; therefore, we ignore the nonlinear part of this equation and concentrate on the simpler case of the linear Poisson equation. This turns out to be equivalent to the Tohline-Kuhn scheme of modified Newtonian gravity as an alternative to dark matter [20-22, 24, 25]. Indeed, on galactic scales, the nonlocal deviation of the gravitational interaction from the inverse-square force law could be responsible for observational data that have been attributed to the presence of dark matter. As a preliminary step, we study some of the implications of the linear nonlocal theory for observations within the solar system.

\section{Appendix A: Integral form of Poisson's equation}

Consider a source density $\rho(\mathbf{x})$ with compact support in a spatial volume $V$ bounded by the surface $S$. Using Eq. (7) and

$$
\nabla^{2} \frac{1}{\left|\mathbf{x}-\mathbf{x}^{\prime}\right|}=-4 \pi \delta\left(\mathbf{x}-\mathbf{x}^{\prime}\right)
$$

in Green's theorem, we find

$$
\Phi(\mathbf{x})=-G \mathcal{I}+\mathcal{S}, \quad \mathbf{x} \in V
$$

while

$$
G \mathcal{I}=\mathcal{S}, \quad \mathbf{x} \notin V
$$

Here

$$
\mathcal{I}=\int_{V} \frac{\rho\left(\mathbf{x}^{\prime}\right)+\rho_{\mathrm{D}}\left(\mathbf{x}^{\prime}\right)}{\left|\mathbf{x}-\mathbf{x}^{\prime}\right|} d^{3} x^{\prime}
$$

and $\mathcal{S}$ is the surface integral

$$
\mathcal{S}=\frac{1}{4 \pi} \oint_{S}\left[\frac{1}{\mathcal{R}} \frac{\partial \Phi}{\partial n^{\prime}}-\Phi\left(\mathbf{x}^{\prime}\right) \frac{\partial}{\partial n^{\prime}}\left(\frac{1}{\mathcal{R}}\right)\right] d S,
$$

where $\mathcal{R}=\left|\mathbf{x}-\mathbf{x}^{\prime}\right|, \partial \Phi / \partial n^{\prime}:=\left(\nabla_{\mathbf{x}^{\prime}} \Phi\right) \cdot \widehat{\mathbf{n}}^{\prime}$ and $\widehat{\mathbf{n}}^{\prime}$ is the unit vector normal to the boundary surface $S$.

In Eq. (A3), where $\mathbf{x} \notin V$, and assuming that $q$ and $\rho$ are continuous functions, the order of integration in $\mathcal{I}$ when Eq. (8) is taken into account - may be interchanged such that

$$
\mathcal{I}=\int_{V} K\left(\mathbf{x}, \mathbf{x}^{\prime}\right) \rho\left(\mathbf{x}^{\prime}\right) d^{3} x^{\prime}
$$

where $K$ is given by

$$
K\left(\mathbf{x}, \mathbf{x}^{\prime}\right)=\frac{1}{\left|\mathbf{x}-\mathbf{x}^{\prime}\right|}+\int_{V} \frac{q\left(\mathbf{y}-\mathbf{x}^{\prime}\right) d^{3} y}{|\mathbf{x}-\mathbf{y}|} .
$$

If $\rho+\rho_{\mathrm{D}}$ is bounded for small $r=|\mathbf{x}|$, falls off as $r^{-(2+\alpha)}$ with $\alpha>0$ for large $r$ and $\Phi \rightarrow 0$ as $r \rightarrow \infty$, then

$$
\Phi(\mathbf{x})=-G \int \frac{\rho\left(\mathbf{x}^{\prime}\right)+\rho_{\mathrm{D}}\left(\mathbf{x}^{\prime}\right)}{\left|\mathbf{x}-\mathbf{x}^{\prime}\right|} d^{3} x^{\prime} .
$$

To satisfy the conditions for Eq. (A8), we note that for a smooth source density $\rho(\mathbf{x})$ with compact support, $\rho_{\mathrm{D}}$ must be finite and $q(\mathbf{x})$ must fall off as $r^{-(2+b)}$ with $b>0$ as $r \rightarrow \infty$, since it is simple to check that

$$
\nabla^{2}\left(\frac{1}{r^{b}}\right)=\frac{b(b-1)}{r^{b+2}} .
$$

\section{Appendix B: Nonuniqueness of $q$}

We consider the time independent, spherically symmetric solutions of the nonlinear equation

$$
\square u=2 u^{2}
$$

in spacetime. Using spherical coordinates, the spherically symmetric, time-independent solutions satisfy the ordinary differential equation

$$
u_{r r}+\frac{2}{r} u_{r}=2 u^{2}
$$

In particular, we wish to determine the solutions that are $C^{\infty}$-flat as $r \rightarrow \infty$.

The change of variables (see $[36,37]$ )

$$
z=r^{2} u, \quad \tau=\ln r
$$

or, equivalently,

$$
z(\tau)=e^{2 \tau} u\left(e^{\tau}\right), \quad u(r)=\frac{1}{r^{2}} z(\ln r),
$$

transforms equation (B2) to the autonomous ordinary differential equation

$$
z^{\prime \prime}-3 z^{\prime}+2 z=2 z^{2}
$$

where the prime signifies differentiation with respect to $\tau$.

In the phase plane (see Figure 1), the corresponding system

$$
z^{\prime}=w, \quad w^{\prime}=3 w-2 z+2 z^{2}
$$

has two rest points $(z, w)=(0,0)$ and $(z, w)=(1,0)$, which correspond to the solutions

$$
u(r)=0, \quad u(r)=1 / r^{2}
$$

of equation (B2); these solutions might also be obtained by inspection of this equation. Both of these solutions and all of their derivatives vanish in the limit as $r \rightarrow \infty$; that is, both are $C^{\infty}$-flat as $r \rightarrow \infty$.

By linearization at the point $(0,0)$ in the phase plane for system (B6), it follows that this point is a hyperbolic source with corresponding spectrum $\{1,2\}$. The rest point $(1,0)$ is a hyperbolic saddle with spectrum $\left\{\frac{1}{2}(3-\sqrt{17}), \frac{1}{2}(3+\sqrt{17})\right\}$. Moreover, the stable manifold 


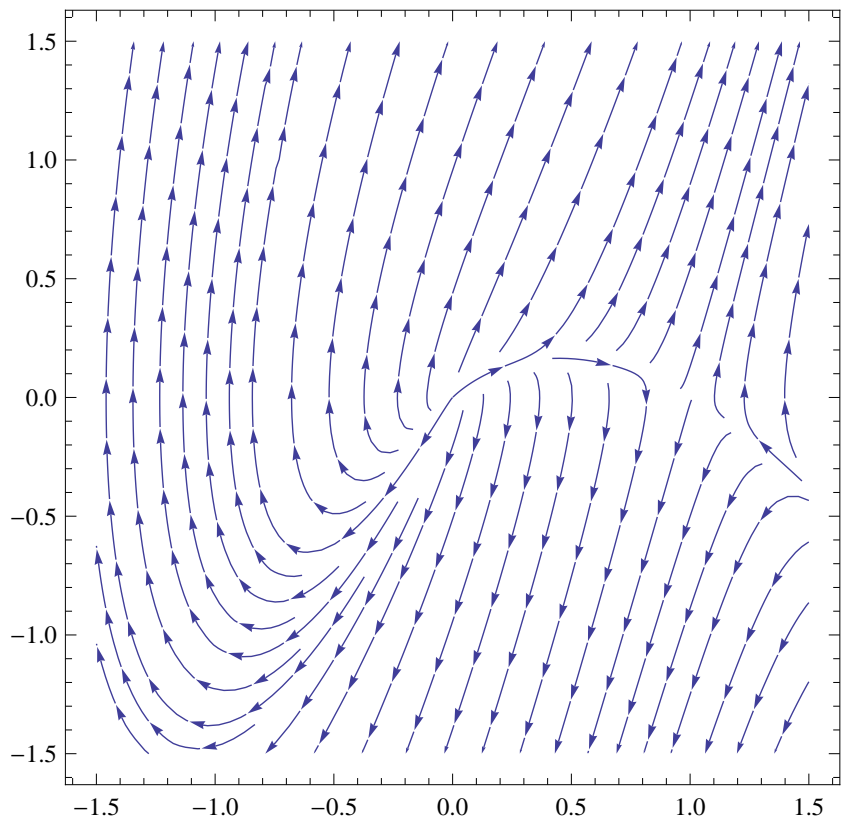

FIG. 1: Phase portrait of system (B6), where $w$ is drawn versus $z$.

of this rest point is tangent to the line with parametrization

$$
s \mapsto\left(\begin{array}{l}
1 \\
0
\end{array}\right)+s\left(\begin{array}{c}
-1 \\
\frac{1}{2}(\sqrt{17}-3)
\end{array}\right) .
$$

A solution of the system whose orbit lies on this stable manifold is asymptotic to a corresponding solution of the linearization on the linearized stable manifold (which is exactly the line in display (B8)). That is, the asymptotic behavior of a solution on the stable manifold is

$$
\tau \mapsto\left(\begin{array}{l}
1 \\
0
\end{array}\right) \pm e^{-\sigma\left(\tau+\tau_{0}\right)}\left(\begin{array}{c}
-1 \\
\frac{1}{2}(\sqrt{17}-3)
\end{array}\right),
$$

where $\sigma=\frac{1}{2}(\sqrt{17}-3)$ and $\tau_{0}$ is an arbitrary real number. In particular, the asymptotic behavior of the first component of a solution on the stable manifold will be

$$
z(\tau) \sim 1 \pm e^{-\sigma\left(\tau-\tau_{0}\right)},
$$

which corresponds to

$$
u(r) \sim \frac{1}{r^{2}} \pm \frac{e^{\sigma \tau_{0}}}{r^{2+\sigma}} .
$$

Because $\sigma>0$, the function $u$ decreases like $1 / r^{2}$ as $r \rightarrow \infty$.

\section{Appendix C: Nonlocal Poincaré gauge theory}

In the traditional approach to general relativity (GR), the invariance of the spacetime manifold under coordinate transformations is emphasized. Thus curvilinear coordinate systems play a dominant role in GR and observers that occupy fixed positions in space in a given coordinate system are accelerated in general. The frame field constructed from linearly independent vectors that are tangent to the curvilinear coordinate lines are called natural or coordinate frames. Such a frame, which is not necessarily orthonormal, is integrable by definition and hence holonomic. The gravitational field equations are second-order local partial differential equations (PDEs) for the spacetime metric in a given system of coordinates.

In the gauge approach to gravity, however, one considers arbitrary frame fields that are in general nonintegrable, that is, anholonomic. Taking advantage of the freedom afforded by the use of anholonomic frames and the associated geometric concepts (such as torsion), the gravitational field equations take the form of firstorder local PDEs [12, 13, 38-40].

It turns out that one can extend the first-order local field equations to nonlocal ones via the introduction of a "constitutive" kernel as in the phenomenological electrodynamics of media. In this way, a nonlocal generalization of Einstein's theory of gravitation becomes possible by starting with the teleparallel equivalent of GR rather than with GR itself $[14,15]$.

In order to be able to construct a nonlocal generalization of Einstein's gravitational theory, the gauge theory of translations was recently employed in $[14,15]$. Now, the gauge theory of translations itself is a somewhat degenerate subcase of the gauge theory of the Poincaré group, the so-called "Poincaré gauge theory of gravity". In turn, the question arises whether the nonlocal generalization of the translational gauge theory can be extended to the Poincaré gauge theory. This is, in fact, the case. We will follow the method used in $[14,15]$ for the translational gauge theory in the more general case of the Poincaré gauge theory. We will use the notation and conventions of [15].

Let the gauge Lagrangian of the underlying RiemannCartan spacetime depend on coframe $e_{i}{ }^{\alpha}$, torsion $T_{i j}{ }^{\alpha}$ and curvature $R_{i j}{ }^{\alpha \beta}=-R_{i j}{ }^{\beta \alpha}$; that is, $\mathcal{L}_{\text {grav }}=$ $\mathcal{L}_{\text {grav }}\left(e_{i}{ }^{\alpha}, T_{i j}{ }^{\alpha}, R_{i j}{ }^{\alpha \beta}\right)$. The matter Lagrangian $\mathcal{L}_{\text {mat }}$, with the matter field(s) $\Psi$, is supposed to be minimally coupled to the geometry. Then the total Lagrangian reads

$\mathcal{L}_{\text {tot }}=\mathcal{L}_{\text {grav }}\left(e_{i}{ }^{\alpha}, T_{i j}{ }^{\alpha}, R_{i j}{ }^{\alpha \beta}\right)+\mathcal{L}_{\text {mat }}\left(e_{i}{ }^{\alpha}, \psi, D_{i} \psi\right)$,

with the independent field variables $e_{i}{ }^{\alpha}$ (coframe), $\Gamma_{i}^{\alpha \beta}=-\Gamma_{i}^{\beta \alpha}$ (Lorentz connection) and $\Psi$ (matter field(s)). With the help of the two excitations

$$
\mathcal{H}^{i j}{ }_{\alpha}=-2 \frac{\partial \mathcal{L}_{\text {grav }}}{\partial T_{i j}{ }^{\alpha}} \quad \text { and } \quad \mathcal{H}^{i j}{ }_{\alpha \beta}=-2 \frac{\partial \mathcal{L}_{\text {grav }}}{\partial R_{i j}{ }^{\alpha \beta}},
$$

the two field equations - the results of the variation of $\mathcal{L}_{\text {tot }}$ with respect to $e_{i}{ }^{\alpha}$ and $\Gamma_{i}{ }^{\alpha \beta}$-can be written as

$$
\begin{aligned}
D_{j} \mathcal{H}^{i j}{ }_{\alpha}-\mathcal{E}_{\alpha}{ }^{i} & =\Sigma_{\alpha}{ }^{i}, \\
D_{j} \mathcal{H}^{i j}{ }_{\alpha \beta}-e^{j}{ }_{[\alpha} \mathcal{H}^{i}{ }_{|j| \beta]} & =\tau_{\alpha \beta}{ }^{i},
\end{aligned}
$$


where $\Sigma_{\alpha}{ }^{i}=\delta \mathcal{L}_{\text {mat }} / \delta e_{i}{ }^{\alpha}$ denotes the canonical energymomentum tensor density of the matter field and $\tau_{\alpha \beta}{ }^{i}=$ $\delta \mathcal{L}_{\text {mat }} / \delta \Gamma_{i}{ }^{\alpha \beta}=-\tau_{\beta \alpha}{ }^{i}$ denotes the corresponding canonical spin (angular momentum) tensor density (note that these definitions differ slightly from the ones in Ref. [16]). The energy-momentum tensor density $\Sigma_{\alpha}{ }^{i}$ should not be confused with the torsion tensor $T_{i j}{ }^{\alpha}$.

In Eq. (C3), the energy-momentum tensor of the gauge fields can be expressed as

$$
\mathcal{E}_{\alpha}{ }^{i}:=e^{i}{ }_{\alpha} \mathcal{L}_{\text {grav }}-\mathcal{H}^{j k}{ }_{\alpha} T_{j k}{ }^{i}-\mathcal{H}^{j k}{ }_{\alpha \beta} R_{j k}{ }^{i \beta} .
$$

On the other hand, for Eq. (C4) the spin of the gauge fields is given by $e^{j}{ }_{[\alpha} \mathcal{H}^{i}{ }_{|j| \beta]}$, which depends only on the translational excitation; thus, it is very simple and we have already substituted it directly into Eq. (C4).

This represents the general framework for the Poincaré gauge theory. We still have to specify the explicit form of the gauge Lagrangian. Following the general scheme of a Yang-Mills theory, we assume that the Lagrangian is local and quadratic in torsion and curvature. We denote the three irreducible pieces of the torsion by ${ }^{(I)} T_{i j}{ }^{\alpha}$, for $I=1,2,3$, and the six irreducible pieces of the curvature by ${ }^{(K)} R_{i j}{ }^{\alpha \beta}$, for $K=1,2, \ldots, 6$; details can be found in $[13,16]$. Then the Lagrangian reads

$$
\begin{aligned}
& \stackrel{\text { Loc }}{\text { grav }}^{\text {gr }}=\frac{1}{2 \kappa} \sqrt{-g}\left[-e^{i}{ }_{\alpha} e^{j}{ }^{\left({ }^{(6)}\right.} R_{i j}{ }^{\alpha \beta}+\Lambda+T^{i j}{ }_{\alpha}\right. \\
& \left.\quad \times \sum_{I=1}^{3} b_{I}{ }^{(I)} T_{i j}{ }^{\alpha}\right]+\frac{\sqrt{-g}}{2 \xi} R^{i j}{ }_{\alpha \beta} \sum_{K=1}^{6} c_{K}{ }^{(K)} R_{i j}{ }^{\alpha \beta},
\end{aligned}
$$

where $\kappa$ is Einstein's gravitational constant, $\Lambda$ is the cosmological constant and $\xi$ is the dimensionless coupling constant of "strong gravity", which is mediated via the propagating Lorentz connection. The constants $b_{I}$ and $c_{K}$ are dimensionless and should be of order unity.

We compute the excitations from Eq. (C6) by partial differentiation according to Eq. (C2):

$$
\begin{aligned}
\mathcal{H}^{i j}{ }_{\alpha} & =\frac{\sqrt{-g}}{\kappa} \sum_{I=1}^{3} b_{I}{ }^{(I)} T^{i j}{ }_{\alpha}, \\
\mathcal{H}^{i j}{ }_{\alpha \beta} & =\frac{\sqrt{-g}}{\kappa} e^{i}{ }_{[\alpha} e^{j}{ }_{\beta]}+\frac{\sqrt{-g}}{\xi} \sum_{K=1}^{6} c_{K}{ }^{(K)} R^{i j}{ }_{\alpha \beta} \\
& =\operatorname{lin}^{i j}{ }_{\alpha \beta}+\mathcal{H}^{\text {qu }}{ }^{i j}{ }_{\alpha \beta} .
\end{aligned}
$$

This is the quadratic local Poincaré gauge theory.

For later purposes, it is convenient to express the Lagrangian in terms of the excitations:

$$
\begin{aligned}
\mathcal{L}_{\text {grav }}= & -\frac{1}{2} \mathcal{H}^{\operatorname{lin}}{ }_{\alpha \beta}{ }^{(6)} R_{i j}{ }^{\alpha \beta}+\frac{\sqrt{-g}}{2 \kappa} \Lambda \\
& -\frac{1}{4} \mathcal{H}^{i j}{ }_{\alpha} T_{i j}{ }^{\alpha}-\frac{1}{4} \stackrel{\mathcal{H}}{ }^{i j}{ }_{\alpha \beta} R_{i j}{ }^{\alpha \beta} .
\end{aligned}
$$

This Lagrangian will also be valid in the nonlocal case. We now generalize the local "constitutive relations" $(\mathrm{C} 7)$ and (C8) to nonlocal ones, again as in [14, 15], by using an unknown scalar kernel $\chi\left(x, x^{\prime}\right)$ and the world function $\Omega$ and its derivatives for transporting tensors from $x^{\prime}$ to $x$ :

$$
\begin{aligned}
& \mathcal{H}^{i j}{ }_{k}(x)=-\frac{\sqrt{-g(x)}}{\kappa} \int U\left(x, x^{\prime}\right) \Omega^{i i^{\prime}} \Omega^{j j^{\prime}} \Omega_{k k^{\prime}} \\
& \times \chi\left(x, x^{\prime}\right) \sum_{I=1}^{3} b_{I}{ }^{(I)} T_{i^{\prime} j^{\prime}} k^{\prime}\left(x^{\prime}\right) \sqrt{-g\left(x^{\prime}\right)} d^{4} x^{\prime}, \\
& \operatorname{lin}_{\mathcal{H}}{ }^{i j}{ }_{k l}(x)=\frac{\sqrt{-g(x)}}{\kappa} \delta_{[k}^{i} \delta_{l]}^{j}, \\
& \stackrel{\mathrm{Hu}}{ }^{i j}{ }_{k l}(x)=\frac{\sqrt{-g(x)}}{\xi} \int U\left(x, x^{\prime}\right) \Omega^{i i^{\prime}} \Omega^{j j^{\prime}} \Omega_{k k^{\prime}} \Omega_{l l^{\prime}} \\
& \times \chi\left(x, x^{\prime}\right) \sum_{K=1}^{6} c_{K}{ }^{(K)} R_{i^{\prime} j^{\prime}}{ }^{\prime} l^{\prime} l^{\prime}\left(x^{\prime}\right) \sqrt{-g\left(x^{\prime}\right)} d^{4} x^{\prime}, \\
& \mathcal{H}^{i j}{ }_{k l}=\stackrel{\operatorname{lin}}{\mathcal{H}}^{i j}{ }_{k l}+\stackrel{\mathrm{H}}{\mathcal{H}}^{i j}{ }_{k l} .
\end{aligned}
$$

The final field equations will not be written down explicitly. We find them as follows: We first substitute Eqs. (C10)-(C13) into (C9) and (C5) and then into the field equations (C3) and (C4); the last step involves the substitution of the new Eq. (C5), after Eq. (C9) is inserted, into Eq. (C3). In this way, we have a set of $16+$ 24 integro-differential equations in terms of the variables $e_{i}^{\alpha}, \Gamma_{i}^{\alpha \beta}$ and $\Psi$.
[1] I.I. Shapiro, "Experimental Tests of the General Theory of Relativity," in General Relativity and Gravitation, edited by A. Held (Plenum, New York, 1980), Vol. 2, pp. 469-489.

[2] C. M. Will, Living Rev. Rel. 9, 3 (2006) [arXiv:grqc/0510072].

[3] C.W.F. Everitt et al., Space Sci. Rev. 148, 53 (2009).
[4] E. G. Adelberger, B. R. Heckel and A. E. Nelson, Ann. Rev. Nucl. Part. Sci. 53, 77 (2003) [arXiv:hep$\mathrm{ph} / 0307284]$.

[5] C. D. Hoyle, D. J. Kapner, B. R. Heckel, E. G. Adelberger, J. H. Gundlach, U. Schmidt and H. E. Swanson, Phys. Rev. D 70, 042004 (2004) [arXiv:hep-ph/0405262].

[6] E. G. Adelberger, B. R. Heckel, S. A. Hoedl, C. D. Hoyle, 
D. J. Kapner and A. Upadhye, Phys. Rev. Lett. 98, 131104 (2007) [arXiv:hep-ph/0611223].

[7] D. J. Kapner, T. S. Cook, E. G. Adelberger, J. H. Gundlach, B. R. Heckel, C. D. Hoyle and H. E. Swanson, Phys. Rev. Lett. 98, 021101 (2007) [arXiv:hep-ph/0611184].

[8] A. Einstein, The Meaning of Relativity (Princeton University Press, 1950), p. 60.

[9] H. Weyl, Space-Time-Matter (Dover, New York, 1952), pp. 176-177.

[10] B. Mashhoon, "Nonlocal theory of accelerated observers," Phys. Rev. A 47, 4498 (1993); B. Mashhoon, "Nonlocal special relativity," Ann. Phys. (Berlin) 17, 705 (2008); B. Mashhoon, "Optics of rotating systems," Phys. Rev. A 79, 062111 (2009).

[11] B. Mashhoon, Ann. Phys. (Berlin) 16, 57 (2007).

[12] P. von der Heyde, Lett. Nuovo Cimento 14, 250 (1975).

[13] F. Gronwald and F. W. Hehl, "On the gauge aspects of gravity," Talk given at International School of Cosmology and Gravitation: 14th Course: Quantum Gravity, Erice, Italy, 11-19 May 1995. In *Erice 1995, Quantum gravity*, pp. 148-198 [arXiv:gr-qc/9602013].

[14] F. W. Hehl and B. Mashhoon, Phys. Lett. B 673, 279 (2009).

[15] F. W. Hehl and B. Mashhoon, Phys. Rev. D 79, 064028 (2009).

[16] F. W. Hehl, J. Nitsch, and P. Von der Heyde, in General Relativity and Gravitation, edited by A. Held (Plenum, New York, 1980), Vol. 1, p. 329; see, in particular, the Appendix.

[17] D. Clowe et al., Astrophys. J. Lett. 648, L109 (2006); D. Clowe, S. W. Randall and M. Markevitch, Nucl. Phys. B (Proc. Suppl.) 173, 28 (2007).

[18] C. Carignan, L. Chemin, W. K. Huchtmeier, and F. J. Lockman, Astrophys. J. Lett. 641, L109 (2006).

[19] P. Salucci et al., Mon. Not. R. Astron. Soc. 378, 41 (2007); G. Gentile, B. Famaey, H.-S. Zhao and P. Salucci, Nature 461, 627 (2009).

[20] J. E. Tohline, in IAU Symposium 100, Internal Kinematics and Dynamics of Galaxies, edited by E. Athanassoula (Reidel, Dordrecht, 1983), p. 205.

[21] J. R. Kuhn and L. Kruglyak, Astrophys. J. 313, 1 (1987).
[22] J. D. Bekenstein, in Second Canadian Conference on General Relativity and Relativistic Astrophysics, A. Coley, C. Dyer and T. Tupper, eds. (World Scientific, Singapore, 1988), p. 68.

[23] R. B. Tully and J. R. Fisher, Astron. \& Astrophys. 54, 661 (1977).

[24] J. D. Bekenstein, Phys. Rev. D 70, 083509 (2004); erratum, Phys. Rev. D 71, 069901(E) (2005) [arXiv:astro$\mathrm{ph} / 0403694]$.

[25] M. Milgrom, Phys. Rev. D 80, 123536 (2009) [arXiv:0912.0790 [gr-qc]].

[26] O. D. Kellogg, Foundations of Potential Theory (Dover, New York, 1953).

[27] J. D. North, The Measure of the Universe (Clarendon Press, Oxford, 1965).

[28] I. S. Gradshteyn and I. M. Ryzhik, Table of Integrals, Series and Products (Academic Press, New York, 1980).

[29] J. L. Synge, Relativity: The Special Theory, 2nd ed. (North-Holland, Amsterdam, 1965).

[30] G. H. Derrick, J. Math. Phys. 5, 1252 (1964); H. Lindblad, Duke Math. J. 72, 503 (1993).

[31] I. Ciufolini, S. Kopeikin, B. Mashhoon and F. Ricci, Phys. Lett. A 308, 101 (2003).

[32] J. M. A. Danby, Fundamentals of Celestial Mechanics, 2nd ed. (Willmann-Bell, Richmond, VA, 1988).

[33] L. D. Landau and E. M. Lifshitz, The Classical Theory of Fields (Pergamon, Oxford, 1971).

[34] H.-J. Schmidt, Phys. Rev. D 78, 023512 (2008).

[35] J. D. Anderson and M. M. Nieto, Astrometric SolarSystem Anomalies, Proceedings of the IAU Symposium 261, to be published [arXiv:0907.2469v2].

[36] L. M. Berkovich, The generalized Emden-Fowler Equation, Symm. Nonlinear Math. Phys. 1, 155 (1997).

[37] H. Goenner and P. Havas, J. Math. Phys. 41, 7029 (2000).

[38] J. A. Schouten, Tensor Analysis for Physicists, 2nd ed., reprinted (Dover, Mineola, NY, 1989).

[39] D. Hartley, Class. Quantum Grav. 12, L103 (1995).

[40] J. M. Nester, Ann. Phys. (Berlin) 19, 45 (2010). 